\title{
Vibroacoustic Models of Air-Core Reactors
}

\author{
Thiago A. Fiorentin \\ Universidade Federal de Santa Catarina - UFSC, Mobility Engineering Center, 89218-035 - Joinville - SC, \\ Brazil
}

\section{Leonardo Ferreira Lopes}

Universidade do Oeste de Santa Catarina - UNOESC, Technological Center, 89600-000 - Joaçaba - SC, Brazil

\section{Olavo Mecias da Silva Junior and Arcanjo Lenzi}

Universidade Federal de Santa Catarina - UFSC, Department of Mechanical Engineering, 88040-900 - Florianópolis - SC, Brazil

(Received 8 January 2015, accepted: 21 October 2015)

The purpose of this paper is to provide an overview of the sound power radiation mechanism of air-core reactors and to describe the method that is used to calculate sound power by using the electrical load. Sound power radiation of an air-core reactor is related to the alternating current harmonics, the mechanical tension stiffness and, most importantly, the breathing mode resonance. An analytical model that is based on electrical loads and mechanical properties of the air-core reactor is developed to calculate radial and axial forces caused by the radial and axial magnetic induction fields. This study employs the hemispherical spreading theory, which is a simple and common method that is used to predict sound propagation. Additionally, a numerical model is proposed. In this, the excitation of the acoustic field that surrounds the reactor is introduced by considering the radial and axial displacements of the reactor's windings, as the windings are subjected to the action of the radial and axial electromagnetic forces. Finally, a comparison is presented between analytical and numerical models and it is observed that the models are correlated.

\section{NOMENCLATURE}

\begin{tabular}{|c|c|}
\hline$B$ & magnetic induction field \\
\hline$B_{\text {radial }}$ & radial magnetic induction field \\
\hline$B_{a x i a l}$ & axial magnetic induction field \\
\hline$B_{\text {avrg,z }}$ & $\begin{array}{l}\text { average magnetic induction field } \\
\text { at } z \text { direction }\end{array}$ \\
\hline$B_{\text {avrg }, x}$ & $\begin{array}{l}\text { average magnetic induction field } \\
\text { at } x \text { direction }\end{array}$ \\
\hline$c_{0}$ & speed of sound in air \\
\hline$d l$ & infinitesimal element \\
\hline$E$ & equivalent Young's modulus \\
\hline$E_{f i b}$ & Young's modulus of the fiberglass \\
\hline$e$ & thickness of the winding \\
\hline$e_{f i b}$ & thickness of the fiberglass \\
\hline$e_{i s o}$ & thickness of the insulation \\
\hline$F$ & electromagnetic force \\
\hline$F_{\text {avrg }, x}$ & average force at $\mathrm{x}$ direction \\
\hline$F_{\text {axial }}$ & axial electromagnetic force \\
\hline$F_{\text {radial }}$ & radial electromagnetic force \\
\hline$F_{Z, a v r g}$ & average force at $\mathrm{z}$ direction \\
\hline$f$ & frequency of the current \\
\hline$G_{x y}$ & shear modulus at plane $x y$ \\
\hline$G_{x z}$ & shear modulus at plane $\mathrm{xz}$ \\
\hline$G_{y z}$ & shear modulus at plane yz \\
\hline$H$ & average height of the winding \\
\hline$h_{w s}$ & height of the reactor without the spiders \\
\hline$I_{e f f}$ & effective current \\
\hline
\end{tabular}

$i$

K

$K_{e q}$

$K_{f i b 1}$

$K_{f i b 2}$

$l$

$l_{m s}$

$\overline{L_{P}}$

$L_{P}$

$L_{W}$

M

N

$n b r$

$p$

$p_{0}$

$R$

$R_{e}$

$R_{i}$

$r$

$r_{s r}$

$S$

$S_{m}$

$S_{W}$

$S_{0}$

$t$

$\nu_{\text {rad }}$

W

$W_{0}$ electrical current

stiffness of a mechanical system

equivalent stiffness

stiffness of fiber layer 1

stiffness of fiber layer 2

height of the material

perimeter of measurement surface

average sound pressure

sound pressure level

sound power level

mass of the winding

number of turns per unit of length

total average number of turns in the winding

sound pressure

reference sound pressure

average radius of the winding

external radius of the winding

internal radius of the winding

distance point to source

distance source-receiver

surface of contact between two materials

surface area of measurement

sound radiating surface

reference area

time

average radial speed of the winding

radiated sound power

reference power 


\begin{tabular}{ll}
\hline \hline$W_{\text {rad }}$ & $\begin{array}{l}\text { radial sound power of the winding } \\
\text { axial sound power of the winding }\end{array}$ \\
$W_{a x i}$ & $\begin{array}{l}\text { distance in } \mathrm{Z} \text { axis of the point } \mathrm{P} \\
z\end{array}$ \\
$\rho_{0}$ & $\begin{array}{l}\text { density of the air } \\
\text { diameter of the conductor without insulating }\end{array}$ \\
$\Phi_{n i}$ & $\begin{array}{l}\text { diameter of the insulated conductor } \\
\Phi\end{array}$ \\
$\mu_{0}$ & constant of proportionality \\
$\omega$ & angular frequency \\
$\sigma$ & radiation efficiency \\
$\Delta R$ & radial displacement of the winding \\
$\Delta H$ & axial displacement of the winding \\
$<\bar{\nu}>\quad$ RMS value of the vibration \\
& velocity over the surface and time \\
$\nu_{x y}$ & Poisson's ratio at plane xy \\
$\nu_{x z}$ & Poisson's ratio at plane $\mathrm{xz}$ \\
$\nu_{y z}$ & Poisson's ratio at plane yz
\end{tabular}

\section{INTRODUCTION}

The population growth and the increasing use of electricity demands the construction of substations for power transmission near major consumption centers. Due to this, the surrounding communities are affected by the noise generated by these stations. ${ }^{1}$ Substation noise is a problem not only because of the high power levels, but also because of the presence of tonal noises that can cause discomfort. Among the main sources of noise in these industrial plants are transformers, capacitors, and air-core reactors.

When considering the impact of audible noise emanating from a high voltage direct current (HVDC) station, the alternating current $(\mathrm{AC})$ filter reactors, and the HVDC smoothing reactor are the main types of air-core reactors that need to be considered. According to the International Council on Large Electric Systems, ${ }^{2}$ the forces resulting from the interaction between the current flow through the reactor and its magnetic induction cause the vibration of reactor surfaces. Some researchers consider that, from the viewpoint of noise generation, vibration amplitude and area of radiating surface determine the sound power generated by air-core reactors. ${ }^{3,4}$

There is a lot interest in determining the forces acting in the radial direction because they induce bending waves on reactor surface, while axial forces excite longitudinal waves. In the case of air-core reactors, the radiation efficiency of bending waves is greater than the longitudinal waves.

This paper describes the mechanism of sound generation in air-core reactors. Two models for calculating the sound power level of reactors are presented: the analytical model estimates the sound power from the radial and axial force created by the axial and radial magnetic field that acts over the reactor and the numerical model uses the radial and axial forces calculated by the analytical model as the excitation of the acoustic field that surrounds the reactor. The analytical and numerical results of a typical configuration of an air-core reactor used in HVDC system are compared with experimental results.

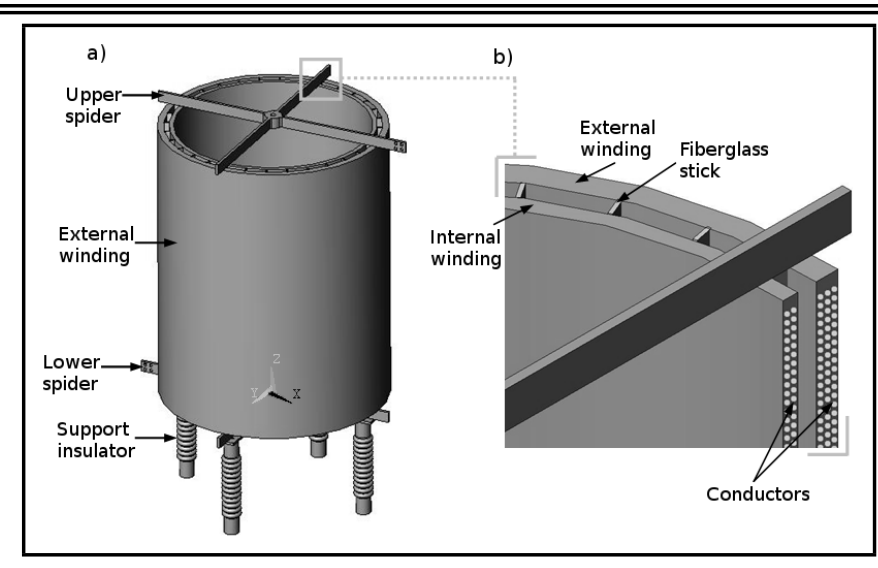

Figure 1. The air-core reactor with: (a) general view and (b) section view.

\section{AIR-CORE REACTORS}

It is common practice to employ air-core reactor technology for different applications in HVDC systems. In such places, reactors have several functions: harmonic filtering on the $\mathrm{AC}$ and DC side, reduction of high frequency noise propagation in the lines, and providing inductive compensation for AC harmonic filters, especially under light load conditions where a certain minimum number of harmonic filters are required to satisfy harmonic performance requirements.

The major construction features of an air-core reactor are illustrated in Fig. 1. The winding of the reactor consists of one or more resin impregnated and encapsulated layers made of insulated aluminum conductors. The concentric layers are connected in parallel by welding their ends to metallic beam structures, called spiders. Both the top and bottom spider are clamped together by several sets of fiberglass ties located along the winding. The packages are radially spaced by circumferentially arranged fiberglass reinforced sticks, which form vertical air ducts for natural convective cooling of the windings.

The noise generated by air-core reactors results mainly from vibration forces caused by the interaction of the current flowing through the winding and its magnetic field. The forces in the winding are proportional to the current multiplied by the magnetic field in the winding, and thus they are proportional to the square of the current. ${ }^{5}$ The forces of interest are primarily those in radial direction, since they create vibrations on the surface that can propagate sound waves in the air.

The acoustic frequency spectrum depends on the load current spectrum of the reactor, and is thus dependent on the reactor application. In the case of single frequency $\mathrm{AC}$ current, the forces oscillate with twice the frequency of the current. However, if the reactor is simultaneously loaded by several currents of different frequencies and to vibration modes at double the electrical frequencies, there are also additional vibration frequencies. This situation is better illustrated by the simplified current spectrum of an AC filter reactor, Fig. 2a, in which the current consists of a component with fundamental frequency $f$ and one harmonic component with harmonic order, $h$. The force acting on the winding of these reactors consists of a static preload and components with frequencies $2 f, f(h-1)$, $f(h+1)$ and $2 f h$, as is shown at Fig. 2b. Only the vibration 


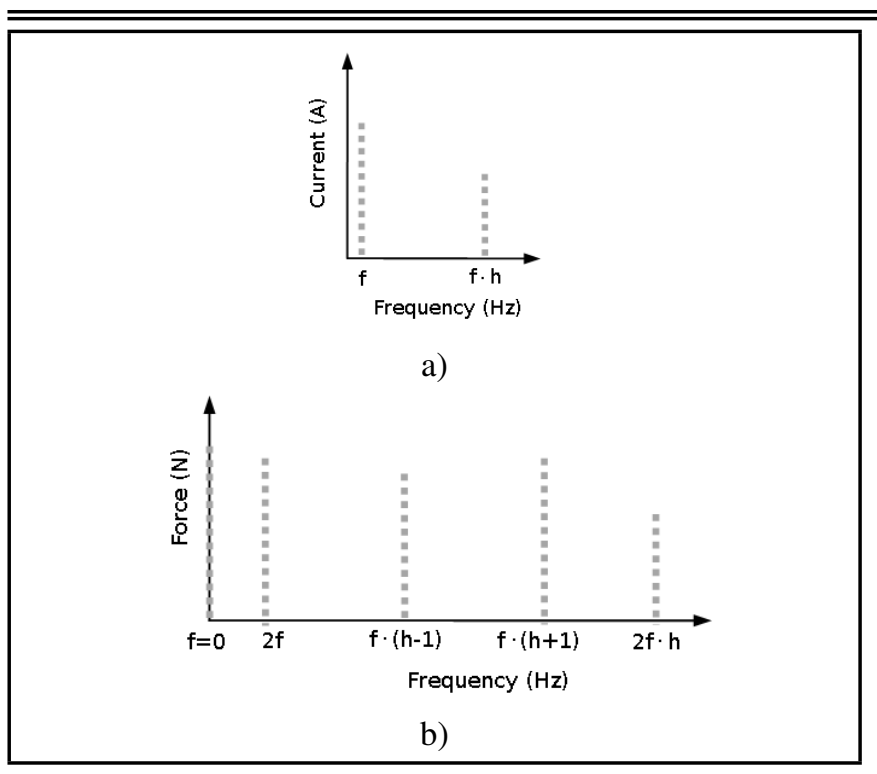

Figure 2. (a) Currents through AC filter reactor and (b) Forces acting on the reactor winding.

force components generate noise, the static preload does not affect the sound power.

It may be concluded that a frequency shift occurs when electrical forces are generated from the electrical load. The number of forces is equal to the square of the number of loads. The acoustic frequency spectrum will therefore significantly increase if the reactor current spectrum includes several harmonic frequencies.

The dynamic behavior of the reactor may be described in terms of vibration modes caused by these electromagnetic forces. Since the oscillating forces are of an almost rotational symmetry, symmetrical modes of the structure coinciding with the shape of the force distribution would expected to be strongly excited. The fundamental modes of the cylindrical reactor structure are:

a) The breathing mode. In this mode the forces are uniformly distributed around the reactor and try alternately to expand and compress the winding in the radial direction or, in other words, the reactor winding is deformed as a cylindrical pressure vessel. This modal frequency essentially depends on the material parameters of the winding and is inversely proportional to the winding diameter. The breathing mode is fully symmetrical and its shape coincides with the distributed exciting electromagnetic force resulting from the axial magnetic field component.

b) The compression mode in the axial direction, where the reactor is symmetrically compressed towards the reactor mid-plane. This mode is excited by the radial magnetic field component.

c) The flexural or bending modes of the winding layers, which is characterized by the number of nodes in circumferential and axial direction. The frequencies of interest for these modes are usually lower than the breathing mode frequency. Although the flexural modes are not of rotational symmetry they become excited by the electromagnetic forces.
Table 1. The geometrical characteristics of the reactor.

\begin{tabular}{|c|c|c|}
\hline $\begin{array}{c}\text { Geometrical characteristics } \\
\text { of the reactor }\end{array}$ & Nomination & Values \\
\hline $\begin{array}{c}\text { Diameter of the conductor } \\
\text { without insulating }\end{array}$ & $\Phi_{n i}$ & $4.79 \times 10^{-3} \mathrm{~m}$ \\
\hline Diameter of the insulated conductor & $\Phi$ & $5.19 \times 10^{-3} \mathrm{~m}$ \\
\hline Average height of the winding & $H$ & $1.20 \mathrm{~m}$ \\
\hline Internal radius of the winding & $R_{i}$ & $5.66 \times 10^{-1} \mathrm{~m}$ \\
\hline External radius of the winding & $R_{e}$ & $5.75 \times 10^{-1} \mathrm{~m}$ \\
\hline Average radius of the winding & $R$ & $5.70 \times 10^{-1} \mathrm{~m}$ \\
\hline Mass of the winding & $M$ & $98 \mathrm{~kg}^{-3} \mathrm{~m}$ \\
\hline Thickness of the winding & $e$ & $9.00 \times 10^{-3} \mathrm{~m}$ \\
\hline Thickness of the insulation & $e_{i s o}$ & $4.00 \times 10^{-4} \mathrm{~m}$ \\
\hline Thickness of the fiberglass layer & $e_{f i b}$ & $3.80 \times 10^{-3} \mathrm{~m}$ \\
\hline Total average number of turns \\
in the winding & $n b r$ & $66.50 \mathrm{turns}$ \\
\hline
\end{tabular}

The vibration amplitude and size of the sound radiating surface of the apparatus essentially determine the sound power. Therefore, the sound emission of an air-core reactor is governed by the magnitude of the winding vibration in the radial direction, since the winding represents the main part of the radiating surface. The contribution of axial winding vibrations and that of other components to the total sound emitted is relatively low.

The results of an air-core reactor, which has the configuration usually found in HVDC stations, are presented in this paper. It has one winding, natural cooling, and its winding is formed by one layer of insulated aluminum conductor. Among the conductors, there are layers of fiberglass with epoxy resin. Other geometrical characteristics of the reactor are shown in Table 1.

\section{SOUND GENERATION MECHANISM}

Alternating currents through the reactor simultaneously produce an electric field due to the electric charges and a magnetic field because of the flowing current. The resulting electromagnetic interaction results in the creation of an electromagnetic force, which causes the vibration of the walls.

\subsection{Analytical Model}

The determination of the expression of the magnetic induction field in all points of the winding involves the solution of complex numerical equations, which does not allow for a simple analytical expression of the field according to the parameters of the system. Therefore, in order to develop an analytical model, some hypothesis are assumed, and the magnetic induction field is broken up in two parts: the radial magnetic induction field $B_{\text {radial }}$, and the axial magnetic induction field $B_{\text {axial }}$. This form will enable the division of the problem in two parts: the radial mode involves the calculation of the axial field, which causes a radial electromagnetic force $F_{\text {radial }}$ and the axial mode, which involves the calculation of the radial field and causes an axial electromagnetic force $F_{\text {axial }}$.

The determination of the expressions of the radial and axial fields will be carried out by the use of the following relations: ${ }^{6}$

a) The Biot-Savart Law expresses the value of the magnetic induction field $\mathbf{B}$ created by an infinitesimal element of 


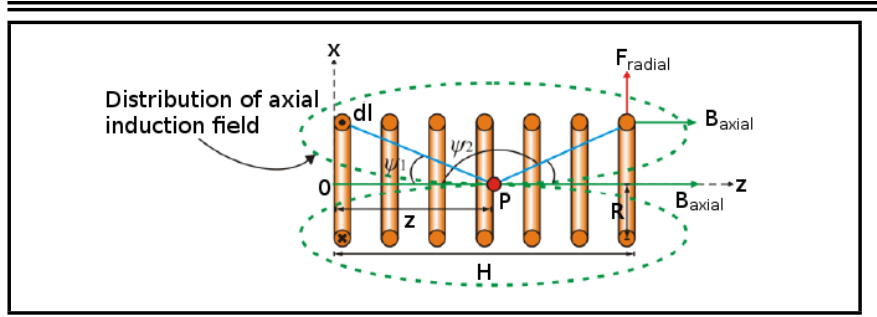

Figure 3. The axial induction field in a point $\mathrm{P}$ of the axis of the winding.

current $\mathbf{d l}$ in a distant point $\mathbf{r}$ of the source.

$$
\mathbf{B}(t)=\frac{\mu_{0}}{4 \pi} i(t) \oint \frac{\mathbf{d} \mathbf{l} x \mathbf{r}_{0}}{r^{3}} ;
$$

where $\mu_{0}$ is the permeability of free space. The small circle on the integral sign indicates that the path of integration is a closed loop. Since the current has a sinusoidal behavior with respect to time, it can be expressed as:

$$
i(t)=\sqrt{2} I_{e f f} \sin (\omega t) ;
$$

where, $I_{\text {eff }}$ is the effective current (A) and $\omega$ is angular frequency of the current ( $\mathrm{rad} / \mathrm{s})$.

b) Ampere's Law provides a method for evaluating $B$ fields when the current distribution has some simplifying features. The law relates the path integral of the magnetic induction field $B$ around a closed loop to the total current $i(t)$ passing through the loop. In mathematical terms, this can be written:

$$
\oint \mathbf{B}(t) \mathbf{d} \mathbf{l}=\mu_{0} i(t)
$$

c) The electromagnetic force equation is defined by:

$$
\mathbf{F}(t)=\oint i(t) \mathbf{d} \mathbf{l} x \mathbf{B}
$$

\subsubsection{Radial mode}

To simplify the development of equations, the value of the axial induction field is calculated on the axis of the winding, as illustrated in Fig. 3. It will be uniformly distributed into the winding and the conductors. The magnetic induction field in a point $P$ of the axis of the winding, Eq. (5), can be estimated by the initial calculation of the field on the axis of one turn using the Biot- Savart Law, which is followed by the extrapolation of the expression to an assembly of $n$ turns: ${ }^{7}$

$$
\begin{aligned}
B(z, t) & =\frac{\sqrt{2} \mu_{0} I_{e f f} N}{2}\left(\frac{z}{\left(R^{2}+z^{2}\right)^{1 / 2}}+\right. \\
& \left.+\frac{(H-z)}{\left(R^{2}+(H-z)^{2}\right)^{1 / 2}}\right) \sin (\omega t) ;
\end{aligned}
$$

where, $\mu_{0}$ is the permeability of free space, $I_{e f f}$ is the effective current, $N$ is the number of turns per unit of length $(N=1 / \Theta), z$ is the distance in $Z$ axis of the point $P, R$ is the average radius of the winding, $H$ is the height of the winding $(H=n b r \cdot \Theta)$, and $\omega$ is the angular frequency of the current.

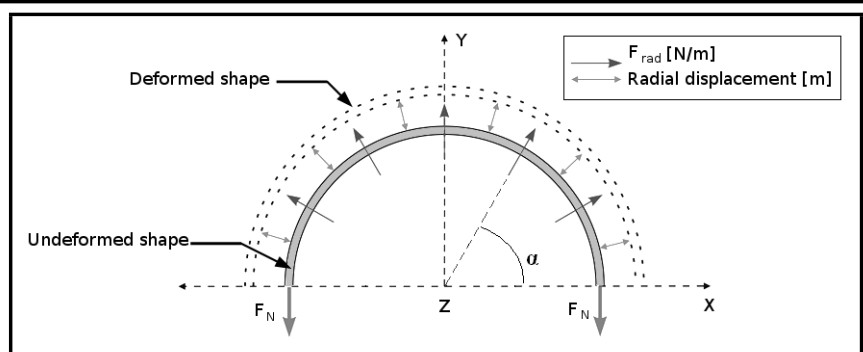

Figure 4. The radial displacement caused by the action of the radial force.

Observe that the axial induction field is maximum in the center of the winding and minimum at the two ends.

To avoid the complex integration of Eq. (5) over the height of the winding, the following calculation of the average axial magnetic induction field, Eq. (6), will be used:

$$
\begin{array}{r}
B_{\text {axial }}(t)=B_{\text {avrg }, z}(t)=\frac{\sqrt{2} \mu_{0} I_{\text {eff }} N}{H}\left(\left(R^{2}+\right.\right. \\
\left.\left.+(H)^{2}\right)^{1 / 2}-R\right) \sin (\omega t) .
\end{array}
$$

Because the turns of the winding are subjected to the average magnetic induction field $B_{\text {axial }}$ (constant in all inner points of the winding), each turn of the winding is subjected to the same radial linear force $F_{\text {radial }}$ determined by Eq. (7).

$$
\begin{array}{r}
F_{\text {radial }}(t)=F_{\text {avr }, x}(t)=\frac{2 \mu_{0} I_{\text {eff }}^{2} N}{H}\left(\left(R^{2}+\right.\right. \\
\left.\left.+(H)^{2}\right)^{1 / 2}-R\right) \sin ^{2}(\omega t) .
\end{array}
$$

The radial force is expressed in $\mathrm{N} / \mathrm{m}$ and has the following properties:

a) The force is unidirectional and repulsive, i.e., no deformation towards the inner part of the winding;

b) The force is proportional to the square of the current, $I_{e f f}$, and proportional to the number of turns per unit of length $N$;

c) The frequency of the average force is twice the frequency of the current.

Figure 4 shows the distribution of the radial force acting over one turn of the winding. $F_{N}$ is the normal force of traction that acts over the thickness of the turn.

Making the vertical balance of the forces that act in $Y$ direction, the following equation for the radial displacement of the winding is:

$$
\Delta R(t)=\frac{2 \mu_{0} I_{e f f}^{2} R^{2}}{n b r \varphi^{3} E e}\left[\left(R^{2}+(n b r \varphi)^{2}\right)^{1 / 2}-R\right] \sin ^{2}(\omega t)
$$

where, $n b r$ is the total average number of turns in the winding, $\varphi$ is the conductor diameter, $e$ is the thickness of the winding, $E$ is an equivalent Young's modulus calculated based on the total area of the winding subjected to the normal force and the total areas of the conductor and fiberglass. Young's modulus of the aluminum and the fiberglass are $7.2 \times 10^{10} \mathrm{~N} / \mathrm{m}^{2}$ and $3.0 \times 10^{10} \mathrm{~N} / \mathrm{m}^{2}$, respectively. 
Deriving the expression of radial displacement with respect to time yields the expression of the average radial speed of the winding as follows:

$$
\begin{array}{r}
\nu_{\text {rad }}(t)=\frac{4 \mu_{0} I_{\text {eff }}^{2} R^{2} \pi f}{n b r \varphi^{3} E e}\left[\left(R^{2}+(n b r \varphi)^{2}\right)^{0.5}-\right. \\
-R] \sin (2 \omega t)
\end{array}
$$

where $f$ is the frequency of the current in hertz.

\subsubsection{Axial mode}

For example, considering the winding formed by four turns located parallel one against each other, as shown in Fig. 5.

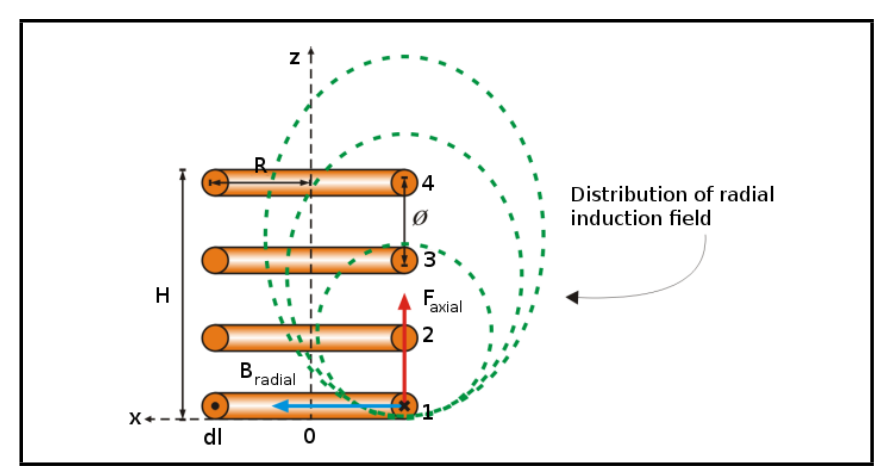

Figure 5. The radial induction field of the first turn of the winding. ${ }^{\dagger}$

The radial induction field that acts over the first turn is equivalent to the sum of the contributions of the fields created by the others turns. The field that acts over the second turn is null because of the cancelation of the induction of the first and third turns. So, it is possible to conclude that the resulting field that acts over the median turn is null. Using these conclusions and Ampere's Law for a linear conductor, the radial induction field in a point located at $z=0, z<H / 2, z=H / 2, z>H / 2$ and $z=H$, may be obtained. These expressions utilize convergent series that have hard solutions. To simplify the analytical calculation procedure, these expressions are reduced to an expression that can be integrated on the half height of the winding in order to obtain the average value of the radial magnetic induction field on both sides. ${ }^{7}$

$$
\begin{array}{r}
B_{\text {radial }}(t)=B_{\text {avrg }, x}(t)= \\
= \pm \frac{\sqrt{2} \mu_{0} I_{\text {eff }}}{4 \pi \varphi} \ln (n b r) \sin (\omega t) \rightarrow(+) \\
\text { when } z<H / 2 \text { or }(-) \text { when } z>H / 2 ; \\
B_{\text {radial }}(t)=B_{\text {avrg }, x}(t)=0 \rightarrow z=H / 2 ;
\end{array}
$$

where, $\varphi$ is the diameter of the conductor and nbr is the total average number of the turns in the winding.

Therefore, consider again that the winding is subject to average radial induction field. Each turn undergoes the same linear

\footnotetext{
${ }^{\dagger}$ For better understanding of the drawing the turns were isolated, but nor-
} mally the distance between them is null.

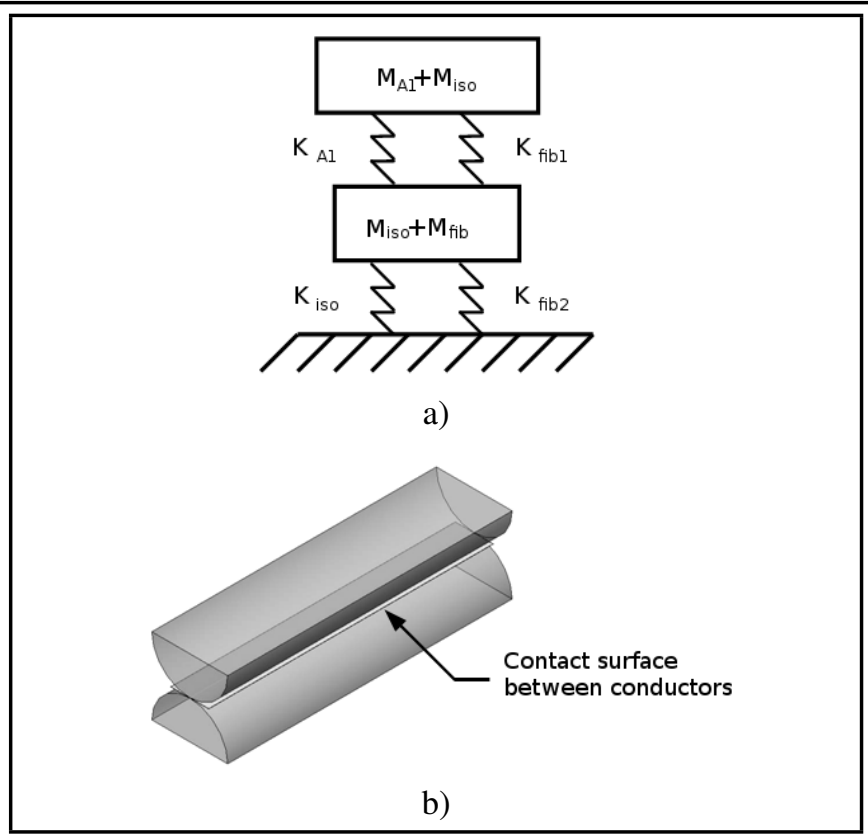

Figure 6. (a) Equivalent stiffness of each turn that forms the winding and (b) Contact surface between materials.

force:

$$
\begin{array}{r}
F_{\text {axial }}(t)=F_{Z, \text { avrg }}(t)= \\
= \pm \frac{\mu_{0} I_{e f f}^{2}}{2 \pi f} \ln (n b r) \sin ^{2}(\omega t) \rightarrow(+) \\
\text { when } z<H / 2 \text { and }(-) \text { when } z>H / 2 ; \\
F_{\text {axial }}(t)=F_{Z, \text { avrg }}(t)=0 \rightarrow z=H / 2 .
\end{array}
$$

The axial force is expressed in $\mathrm{N} / \mathrm{m}$ and has the following properties:

a) The force is proportional to the square of the current $I_{e f f}$.

b) The frequency of the average force is twice the frequency of the current.

c) The distribution of the axial force compresses the winding.

The axial force compresses the winding, therefore in this mode of deformation the winding can be seen as a mass-springmass-spring assembly, as shown in Fig. 6a. The first mass consists of the sum of the mass of the conductor and fiberglass while the second mass corresponds to the sum of the mass of the insulator and fiberglass.

The general expression that defines the stiffness $K$ of a mechanical system is:

$$
K=\frac{E S}{l} .
$$

In this case, $l$ corresponds to the height of the material, $S$ indicates the contact surface between two materials, and $E$ is Young's modulus of the material. The following stiffness $K_{A l}$, $K_{f i b 1}, K_{i s o}$, and $K_{f i b 2}$ will exist for one turn.

The contact surface between conductor and insulator is weaker than the contact surface between fiberglass layers, so the stiffness $K_{A l}$ and $K_{\text {iso }}$ can be neglected when compared 
to the stiffness $K_{f i b 1}$ and $K_{f i b 2}$. Once the two springs are in series, the equivalent stiffness of the system is:

$$
K_{e q}=\frac{K_{f i b 1} K_{f i b 2}}{K_{f i b 1}+K_{f i b 2}} .
$$

Young's modulus of the material that forms the turn can be obtained by replacing the expression of the equivalent stiffness in Eq. (14), and assumes that $l$ is equal to the diameter of the turn.

$$
E=\frac{K_{e q} \varphi}{2 \pi e R} .
$$

It may be observed that the value of Young's modulus for the radial mode and axial mode are different.

As in radial mode, using the definition for Young's modulus, the expression for the axial displacement of the winding $\Delta H$ can be obtained:

$$
\Delta H(t)=\frac{\mu_{0} I_{\text {eff }}^{2} n b r}{2 \pi E_{f i b} e_{f i b}} \ln (n b r) \sin ^{2}(\omega t) ;
$$

where, $E_{f i b}$ and $e_{f i b}$ are respectively Young's modulus and the thickness of the fiberglass.

Deriving the expression of the axial displacement in respect to time, the expression of the average axial speed of the winding can be obtained:

$$
\nu_{a x i}(t)=\frac{f \mu_{0} I_{e f f}^{2} n b r}{E_{f i b} e_{f i b}} \ln (n b r) \sin (2 \omega t) .
$$

\subsubsection{Acoustic model}

According to some research, the equation that defines the radiated sound power is: ${ }^{8}$

$$
W=\rho_{0} c_{0} S_{W} \sigma<\bar{\nu}>^{2} ;
$$

where, $W$ is the radiated sound power in Watts, $\rho_{0}$ is the density of the air in $\mathrm{kg} / \mathrm{m}^{3}, c_{0}$ is the speed of sound in air in $\mathrm{m} / \mathrm{s}$, $S_{W}$ is the sound radiating surface in $\mathrm{m}^{2}, \sigma$ is the radiation efficiency, and $\nu$ is the RMS value of the vibration velocity in $\mathrm{m} / \mathrm{s}$ over the surface $(<>)$ and time $(-)$.

For the reactor, in the radial direction the internal and external surfaces are responsible for sound generation $S_{W \text { rad }}=$ $4 \pi H R$. In the axial direction the surface responsible for radiation is the cross sectional area of the winding $S_{W a x i}=e 2 \pi R$. The radiation efficiency depends on the frequency, geometri$\mathrm{cal}$, and structural properties of the component. The value established for the radiation efficiency is multiplied by a correction factor to take into account all approximations made on the analytical model, neglected internal deformation, dissipation etc.

Using the considerations above, the radial and axial sound power of the winding are respectively:

$$
\begin{array}{r}
W_{\text {rad }}=32 \rho_{0} c_{0} \pi^{3} \mu_{0}^{2} \sigma \frac{I_{\text {eff }}^{4} R^{5} f^{2}}{E^{2} e^{2} \varphi^{5} n b r} . \\
\cdot\left[\left(R^{2}+(n b r \varphi)^{2}\right)^{1 / 2}-R\right]^{2} ;
\end{array}
$$

$$
\begin{aligned}
W_{a x i}= & \rho_{0} c_{0} \pi \mu_{0}^{2} \sigma \frac{I_{e f f}^{4} f^{2} n b r^{2} R}{E_{f i b}^{2} e_{f i b}^{2}} . \\
& \cdot[\ln (n b r)]^{2}\left(e_{f i b}+4 \varphi\right) .
\end{aligned}
$$

Therefore, the sound power level generated by the reactor in $\mathrm{dB}$ can be expressed by the following expression:

$$
L_{W}=10 \log _{10}\left(\frac{W_{\text {rad }}+W_{a x i}}{10^{-12}}\right) .
$$

The acoustic pressure emitted by the winding in a specific point where the receiver is found depends on the comparison between the coordinates of the receiver and the dimension of the source. When the distance source-receiver $r_{s r}$ is large compared to dimensions of the source $\left(r_{s r} / H>10\right)$, the reactor is compared with a spherical source. As in most of the times the reactor is installed near the ground, the sound waves are reflected by the ground ${ }^{\ddagger}$. Therefore, the reactor is comparable with a half-spherical source. The acoustic energy of internal surfaces and the power of axial modes take part in the acoustic pressure equation:

$$
p=\sqrt{\frac{\rho_{0} c_{0}\left(W_{r a d}+W_{a x i}\right)}{2 \pi r_{s r}^{2}}} ;
$$

where, $r_{s r}$ is the distance from the receptor to the center of the winding.

When $r_{s r} / H<10$, the reactor is comparable to a cylindrical source. At such distances, the participation of the noise generated by the interior wall can be neglected in front of that coming from external surface. The noise created by the axial mode may also be neglected, since its direction is parallel to the axis of the reactor.

$$
p=\sqrt{\frac{\rho_{0} c_{0} W_{r a d}}{4 \pi\left(r_{s r}+R_{e}\right) H}} ;
$$

where $R_{e}$ is the external radius of the winding.

The sound pressure level $L_{P}$, a quantity that varies according to the environment in which the source is, can be mathematically defined as:

$$
L_{P}=10 \log \left(\frac{p^{2}}{p_{0}^{2}}\right) ;
$$

where $p$ corresponds to the sound pressure in $\mathrm{Pa}$ and $p_{0}$ is $20 \times 10^{-6} \mathrm{~Pa}$.

Sometimes, the noise created by the axial mode may not be neglected. This occurs, for example, when the reactor's dimensions have a considerable axial area to radiate the sound.

\subsubsection{Analytical results}

Considering the geometrical properties presented at Table 1 and the equations explained in sections 3.1.1, 3.1.2, and 3.1.3 it is possible to predict the sound power level generated by this air- core reactor. For the calculations it is supposed that the

\footnotetext{
† The ground is supposed to be a perfectly reflective surface, without absorption.
} 


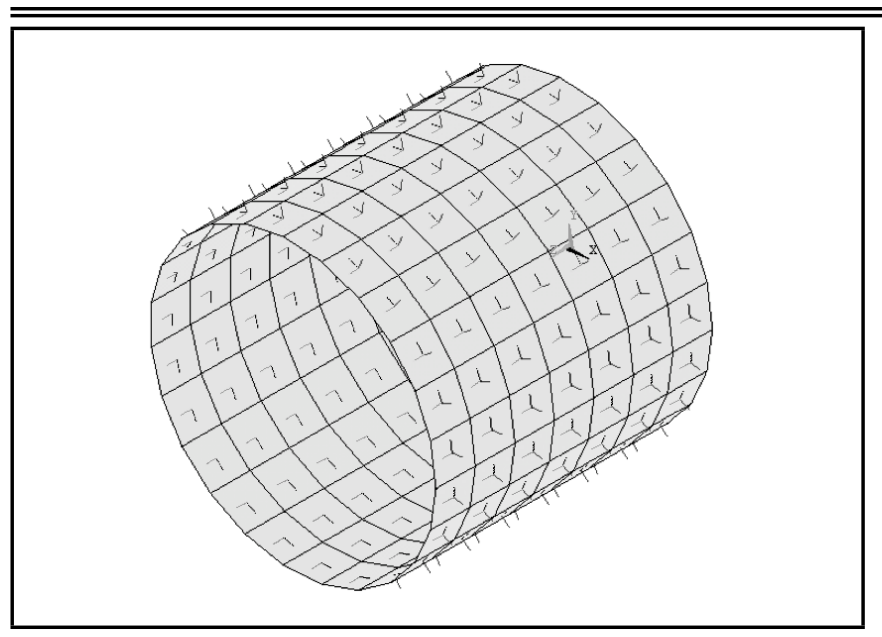

Figure 7. The mesh used for the structural analysis.

reactor is loaded with a single AC current of 300 Amps and a frequency of $60 \mathrm{~Hz}$ and the main answer will be at $120 \mathrm{~Hz}$.

Young's modulus of the aluminum and the fiberglass are $7.2 \times 10^{10} \mathrm{~N} / \mathrm{m}^{2}$ and $3.0 \times 10^{10} \mathrm{~N} / \mathrm{m}^{2}$, respectively. If the ratio $r_{s r} / H<10$, the reactor is comparable to a cylindrical source, then it is possible to use Eq. (24). The established value for the radiation efficiency is 0.25 .

The sound power level calculated to this equipment was $74.3 \mathrm{~dB}$ and the sound pressure level estimated was $58.7 \mathrm{~dB}$. In the next sections these values will be compared with numerical analysis and values determined experimentally.

\subsection{Numerical Analysis}

The numerical models are developed using the finite elements method. The first step of the numeric modeling was to build the geometry corresponding to the reactor analyzed in this research and mesh it. The mesh used for the structural analysis was constructed using the software Ansys 12.1. ${ }^{9}$ The type of the element used was shell 63. This element is defined by four nodes, four thicknesses, elastic foundation stiffness, and orthotropic material properties. Adding to that, the element has both bending and membrane capabilities. Both in-plane and normal loads are permitted. The element has six degrees of freedom at each node with translations in the nodal $x, y$, and $z$ directions and rotations about the nodal $x, y$, and $z$-axes. According to the frequency of interest, the mesh was divided in 22 elements in circumferential direction and 7 elements in axial direction, see Fig. 7.

The analytical forces $F_{\text {axial }}$ and $F_{\text {radial }}$ calculated by analytical model were used as boundary conditions of the structural numerical model. The axial is applied above and below the mid height. The radial force is decomposed into $x$ and $y$ components and applied in all nodes of the model. The boundary conditions are shown in Fig. 8.

The mechanical properties defined for the structural model were: Young's modulus of the fiberglass (10 GPa) for axial and radial directions, and Young's modulus obtained experimentally (30 GPa) for circumferential direction. Shear modulus $G_{x y}(26.7 \mathrm{GPa})$ and $G_{x z}=G_{y z}(1.56 \mathrm{GPa})$ were obtained experimentally. Poisson's ratio $\nu_{x y}$ equals that of the aluminum

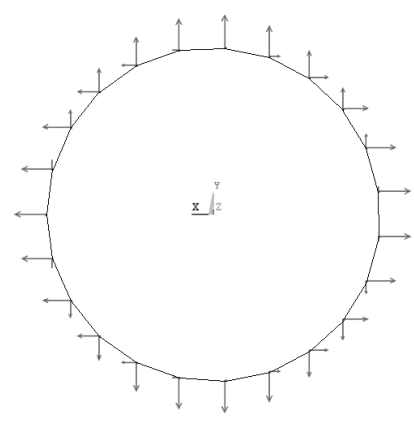

a)

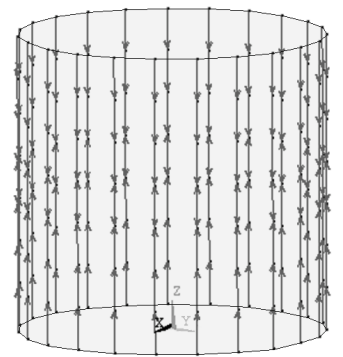

b)

Figure 8. The boundary conditions: (a) radial force decomposed into $x$ and $y$ directions and (b) the axial force.

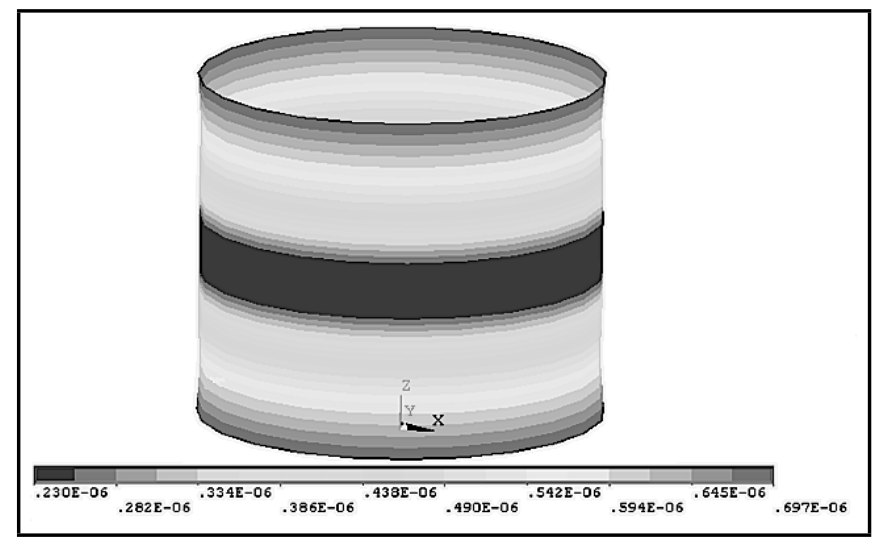

Figure 9. The structural displacement at $120 \mathrm{~Hz}$.

(0.25), $\nu_{x z}=\nu_{y z}$ is the same as the fiberglass (0.034). The average density was calculated, based on the area occupied by the aluminum and the fiberglass in respect to the total area of the reactor, to be $2,362 \mathrm{~kg} / \mathrm{m}^{3}$.

For the calculations the reactor is supposed to be loaded with a single AC current of $300 \mathrm{Amps}$ and frequency of $60 \mathrm{~Hz}$. According to Section 2, the main answer will be at $120 \mathrm{~Hz}$. The software Ansys enables the calculation of harmonic solution for the frequency of interest, Fig. 9.

The acoustic numerical model was developed to calculate the sound power level radiated by the reactor. It was developed using Boundary Element Method (BEM) available in the software Virtual.Lab 11. ${ }^{10}$ The mesh discretization was the same of that in the structural model. The displacements on nodes calculated in the earlier step were used for excitation in the acoustic field. In the software this boundary condition was made by the insertion of a vibrant panel. A field point mesh was created $1.0 \mathrm{~m}$ away from the vibration panel to obtain the 


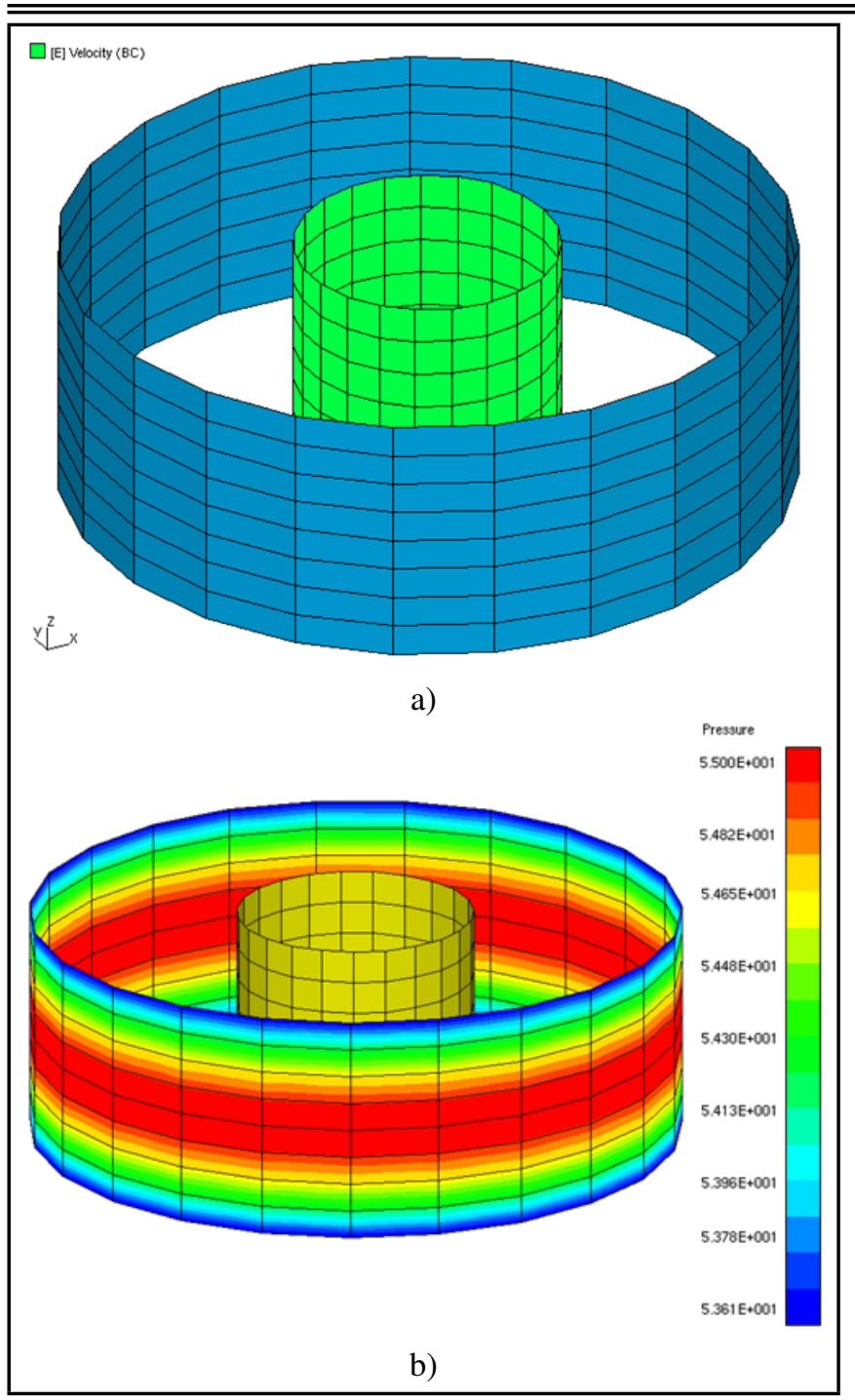

Figure 10. (a) Vibrating panel and field point mesh and (b) the acoustic pressure at $1.0 \mathrm{~m}$ from vibrating panel.

pressure results. The acoustic model can be analyzed through Fig. 10a. The numerical solution enables the determination of the sound level pressure in all nodes of the mesh. The value of sound pressure $1.0 \mathrm{~m}$ from vibrating panel is $55 \mathrm{~dB}$, Fig. $10 \mathrm{~b}$. The sound power level can be evaluated through this value: $69 \mathrm{~dB}$.

\section{EXPERIMENTAL MEASUREMENTS AND DISCUSSION}

The sound power $W$ is the total sound energy emitted by a source per unit of time. To express that greatness on a scale that has a better correlation with human hearing, the sound power level $L_{W}$, which is related to the sound power, is given by the following equation:

$$
L_{W}=10 \log \left(\frac{W}{W_{0}}\right)
$$

where $W$ is the sound power of the source in Watts and $W_{0}$ is the reference power $1 \times 10^{-12}$ Watts. The $L_{W}$ unit is $\mathrm{dB}$. Using the concept of sound intensity, the equation that defines
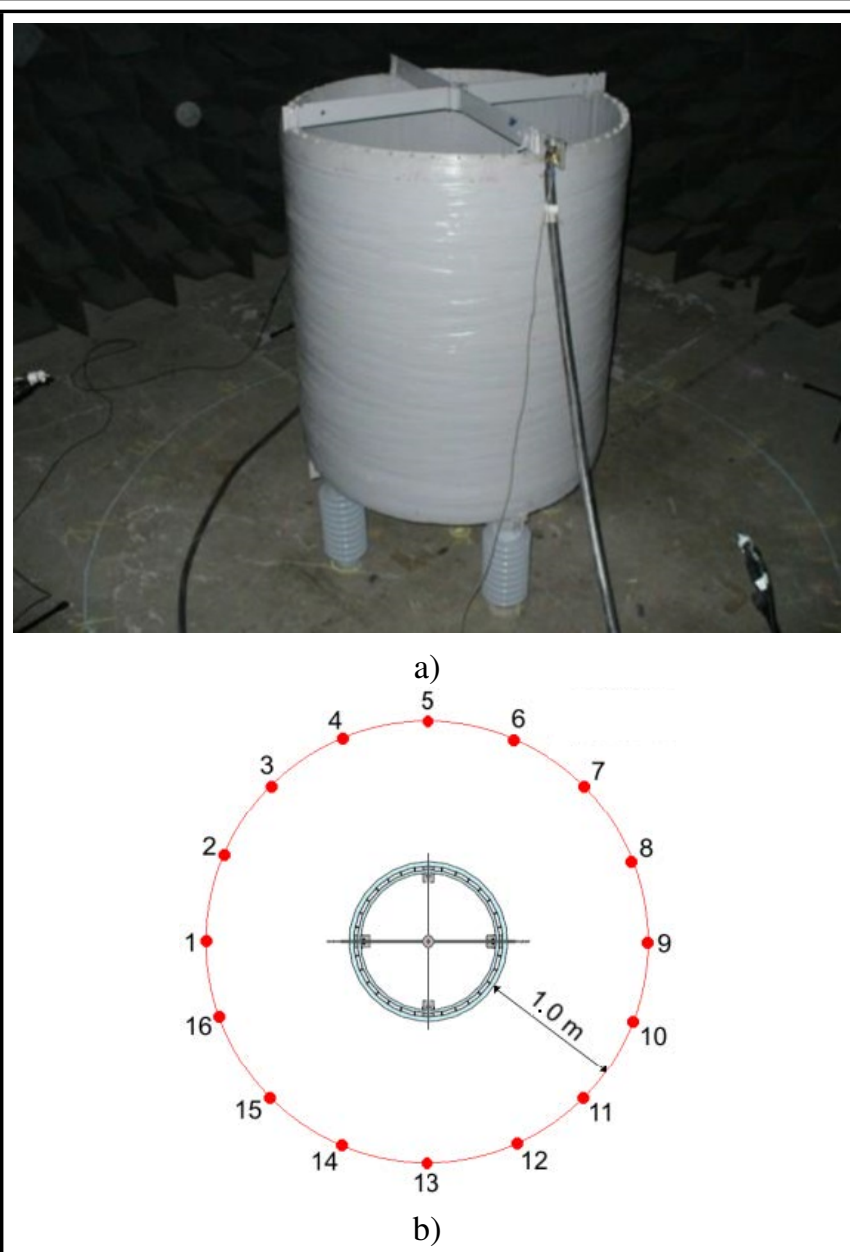

Figure 11. (a) The air-core reactor inside the hemi-anechoic chamber and (b) the circumference with the measurement positions.

de sound power level is rewritten as follows:

$$
L_{W}=\overline{L_{P}}+10 \log \left(\frac{S_{m}}{S_{0}}\right) .
$$

The average value of sound pressure level in $\mathrm{dB}$ is $L_{P}$, the reference area is $S_{0}, 1 \mathrm{~m}^{2}$, and $S_{m}$ is the surface area of measurement in $\mathrm{m}^{2}$. In the case of reactors, the technical standard IEC $60076-10^{11}$ specifies that the microphones must be positioned $1.0 \mathrm{~m}$ from its surface and the surface area of measurement should be calculated by the equation:

$$
S_{m}=\left(h_{w s}+1\right) l_{m s}
$$

where $h_{w s}$ corresponds to the height of the reactor without the spiders and $l_{m s}$ is the perimeter of measurement surface. The sound pressure level $L_{P}$, a quantity that varies according to the environment, is determined mathematically at Eq. (25).

According to standard procedure, air-core reactors with a height less than $2.5 \mathrm{~m}$ must be measured at the half height of the reactor for sound pressure levels. For this height, sixteen measurement positions were defined over an imaginary circumference with the center coincident with the equipment center, according to Fig. 11b. The measurements were performed in the hemi- anechoic chamber, Fig. 11a.

To do the measurements the reactor was loaded with a single $\mathrm{AC}$ current of $300 \mathrm{Amps}$ and a frequency of $60 \mathrm{~Hz}$, the main 


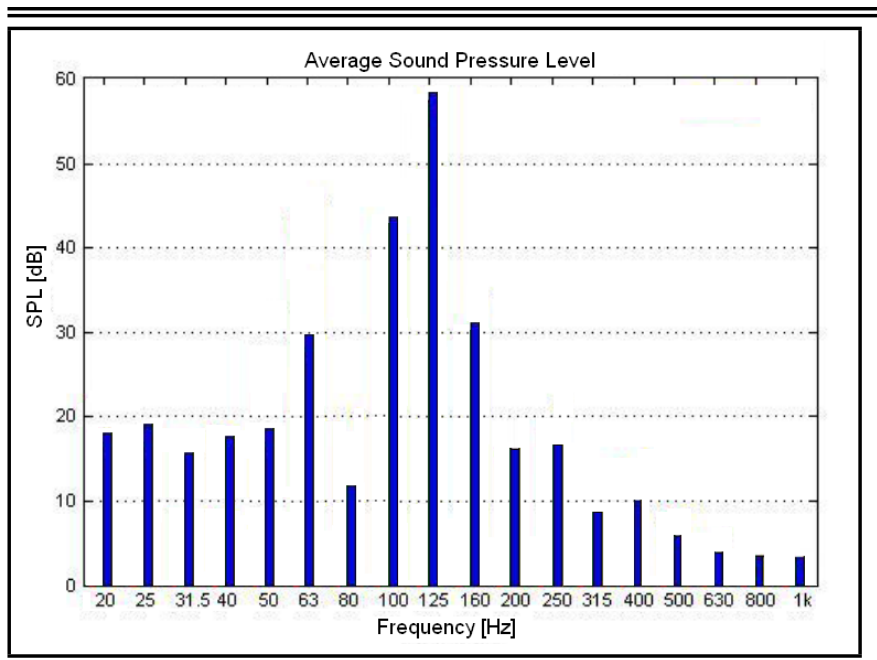

Figure 12. The average sound pressure level measured inside the anechoic chamber.

Table 2. The geometrical characteristics of the reactor.

\begin{tabular}{||c|c|c|c|c|c|}
\hline \multicolumn{2}{|c|}{ Analytical Results } & \multicolumn{2}{c|}{ Numerical Results } & \multicolumn{2}{c|}{ Experimental Results } \\
\hline$\overline{L_{P}}$ & $L_{W}$ & $\overline{L_{P}}$ & $L_{W}$ & $\overline{L_{P}}$ & $L_{W}$ \\
\hline $58.7 \mathrm{~dB}$ & $74.3 \mathrm{~dB}$ & $55.0 \mathrm{~dB}$ & $68.4 \mathrm{~dB}$ & $59.7 \mathrm{~dB}$ & $73.1 \mathrm{~dB}$ \\
\hline
\end{tabular}

answer was $120 \mathrm{~Hz}$. At Figure 12, the average sound pressure level measured inside the hemi-anechoic chamber is presented. The sound pressure level $1.0 \mathrm{~m}$ from reactor was $59.7 \mathrm{~dB}$ and the sound power level was $73.1 \mathrm{~dB}$.

Table 2 compares the results obtained analytically, numerically and experimentally. There is a strong correlation between analytical and experimental results. Comparing numerical and experimental results yields a difference around $4 \mathrm{~dB}$. Considering the many assumptions, these values are reasonable.

\section{CONCLUSIONS}

This paper presents two relatively simple models for the evaluation of the sound power level of air-core reactors. First, the reactor is modeled as a cylinder with axial and radial displacement, and the total sound power calculated as the sum of axial and radial sound power. The developed model is general so that various air-core configurations can be applied. Second, analytical expressions are used as excitation of a structural finite element model. The results obtained through this structural model are used as boundary conditions for the acoustic boundary element model. This approach is most interesting for air-core reactors that have more than one winding.

The experimental results presented enable the identification of frequencies in which the sound power level is larger. In this region of the frequency spectrum, the vibratory energy is sufficiently high to generate relevant noise, depreciating the product.

The analytical results of the sound power level show good agreement with experimental results, thus demonstrating that the analytical model can be useful to calculate the sound pressure generated by air-core reactors. The comparison between experimental and numerical results present some differences. They are explained by the assumptions in the numerical models.
The analytical approach is simple and efficient which allows using it for conducting sensitivity or optimization studies during the design stages.

\section{ACKNOWLEDGEMENTS}

The generous financial support from $\mathrm{CNPq}$ - Conselho Nacional de Desenvolvimento Científico e Tecnológico is highly appreciated.

\section{REFERENCES}

1 Ver I. L., Andersen D. W. Field study of sound radiation by power transformers. IEEE Transactions on Power Apparatus and Systems, 100 (7), 3513-3524. (1981). http://dx.doi.org/10.1109/mper.1981.5511716

2 International Council on Large Electric Systems. Wg 14.26: HVDC stations audible noise. Sweden, 99, (2001). http://dx.doi.org/10.3403/30162952u

3 Smede H., Johansson C. G., Winroth O., Schutt H. P. Design of HVDC converter stations with respect to audible noise requirements. IEEE Transactions on Power Delivery, 10 (2), 747-758, (1995). http://dx.doi.org/10.1109/61.400856

4 Lilien J.L. Acoustic noise generated by air power reactor in open-air substations, European Transaction on Electrical Power, 16, 297-310, (2006). http://dx.doi.org/10.1002/etep.88

${ }^{5}$ Hagiwara S., Hori Y., Suzuki Y., and Obata T. Vibration analysis of a large capacity shunt reactor, IEEE Transaction on Power Apparatus and Systems, 2 (3), 737-745, (1982). http://dx.doi.org/10.1109/tpas.1982.317289

6 Halliday D., Resnick R., Walker J. Fundamentals of Physics, 6th. Hoboken, N. J.: Wiley, (2011).

7 Lopes L. F. Modelo vibroacústico de reatores elétricos com núcleo de ar. Doctoral Thesis, Universidade Federal de Santa Catarina, (2011).

8 Blackstock D. T. Fundamentals Of Physical Acoustics. Ed. John Wiley \& Sons, Inc., New York, USA, (2000).

9 ANSYS, Inc., ANSYS 12.1 Help. ANSYS Mechanical APDL, United States of America, (2009).

10 LMS Virtual.Lab Online Help home page. Virtual.Lab Rev 11. Belgium, (2012).

11 International Electrotechnical Comission. International Standard IEC 60076-10: Power transformers - Part 10: Determination of sound levels. 1-35, (2001). http://dx.doi.org/10.3403/02351228u 